\title{
A Statewide Medication Management System: Health Information Exchange to Support Drug Therapy Optimization by Pharmacists across the Continuum of Care
}

\author{
Karen Pellegrin ${ }^{1}$ Francis Chan ${ }^{2}$ Natalie Pagoria ${ }^{2}$ Sheena Jolson-Oakes ${ }^{3}$ Reece Uyeno ${ }^{1}$ \\ Andrew Levin ${ }^{1}$ \\ ${ }^{1}$ The Daniel K. Inouye College of Pharmacy, University of Hawaii at Hilo, \\ Hilo, Hawaii, United States \\ ${ }^{2}$ Hawaii Health Information Exchange, Honolulu, Hawaii, United States \\ ${ }^{3}$ Maui Clinic Pharmacy, Kahului, Hawaii, United States \\ Address for correspondence Karen Pellegrin, MBA, PhD, University of \\ Hawaii at Hilo, 34 Rainbow Drive, Hilo, Hawaii 96720, United States \\ (e-mail: karen3@hawaii.edu). \\ Appl Clin Inform 2018;9:1-10.
}

\section{Abstract}

Keywords

- drug therapy optimization

- pharmacists

- medication reconciliation

- health information exchange

- health information technology
Background While evidence generally supports the use of medication management technology, systems are typically implemented and evaluated piecemeal rather than as part of a comprehensive model for medication management. Systems to support drug therapy optimization, increasingly a key role of pharmacists in our healthcare system, have not yet been reported.

Objective Our objective is to describe the design, implementation, and use of health information technology to support the hospital and community pharmacists' management of medications for high-risk patients statewide in the "Pharm2Pharm" model of care. Our aims were to make it easier for the pharmacists to access information needed to identify and resolve drug therapy problems using best practices for medication management and communicate with other members of the care team.

Methods The pharmacist's roles and the medication management processes guided the design of the supporting technology, which was implemented after the Pharm2Pharm model was launched and the pharmacists' technology needs were assessed. Priorities for technology included sending care transition documents from hospital to community pharmacist securely and efficiently, access to medical records, including medications and laboratory results, documentation, and patient tracking. Implementation and use of the technology were documented.

Results Communications, medication management, and population management solutions were implemented to support the Pharm2Pharm model. The pharmacists delivering services through this model adopted and meaningfully used this technology to support their work.

Conclusion Implementing technology with value outside of the Pharm2Pharm model was a strategic approach to investment. This work emphasizes the importance of shifting the focus of technology from supporting a specific piece of the medication management process to supporting the goal of optimizing medication regimens. Health information exchange systems can provide important technology needed to integrate pharmacists into care teams as they are deployed to improve patient outcomes. received

June 29, 2017

accepted after revision

November 10, 2017

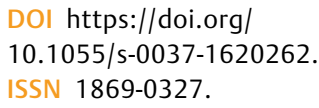

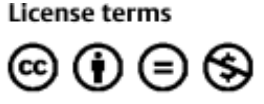




\section{Background and Significance}

Health information technology (IT) in support of medication management has focused primarily on physician use. This is not surprising given that physicians have been a primary target of federal incentives to adopt electronic health records and that generating and transmitting prescriptions electronically has been a key Meaningful Use objective under the CMS Electronic Health Record Incentive Program. ${ }^{1}$ It is estimated that meaningful use of health IT in hospitals was associated with 67,000 fewer adverse drug events from 2010 to 2013. ${ }^{2}$ Meaningful use incentives have resulted in a dramatic increase in the use of e-prescribing and of medication history technology in ambulatory care settings since 2007, with smaller practices more likely to use these technologies than larger practices. ${ }^{3}$ These and other systems are typically implemented and evaluated piecemeal rather than as part of a comprehensive model for medication management. Systems to support drug therapy optimization, increasingly a key role of pharmacists in our healthcare system, have not yet been reported.

While additional research is needed, existing evidence generally supports the use of medication management technology. In an analysis of 87 randomized controlled trials of health IT supporting one or more medication management processes, the majority of studies (74) focused on prescribing and 38 on monitoring. ${ }^{4}$ Almost half of the studies found improvements in medication processes, but less than half of the studies that examined clinical outcomes showed improvements. The three studies with cost as a primary outcome show significant reductions in cost associated with the health IT. A more recent review of electronic tools to support medication reconciliation indicated that future research is needed to determine the effects of these tools on healthcare quality. ${ }^{5}$ The one randomized controlled trial of such an e-tool, combined with redesign of the medication reconciliation process, showed a $28 \%$ reduction in unintentional medication discrepancies with potential for harm, and the intervention effect was greater among high-risk patients. ${ }^{6}$ Similarly, a systematic review found that studies of drug-drug interaction technologies were generally effective, though very few were rigorously designed or measured clinical outcomes. ${ }^{7}$

While the traditional role of the pharmacist is dispensing medications, pharmacists are increasingly serving in clinical roles as members of patient care teams ${ }^{8}$ though are not included as eligible providers under Meaningful Use incentives. Although pharmacists frequently use and document in electronic medical records, there has been little focus on developing health IT to support their workflow in optimizing medication regimens and communicating with other members of the care team. ${ }^{9}$ As research continues to document the positive impact of pharmacists' clinical services across settings ${ }^{10}$ and among older adults ${ }^{11}$ who are at greatest risk of medication problems, ${ }^{12}$ there is increasing justification for investing in technology to make their work more efficient and effective.

The "Pharm2Pharm" program, funded by the CMS Innovation Center, was an ideal opportunity to invest in such technology. This Round One Health Care Innovation Award funded the pharmacists, training for the pharmacists, project management, program evaluation, and some of the technology described in this article during the 4-year award period. This pharmacist-led model targets high-risk inpatients and provides medication management services from hospital to home for up to a year postdischarge. ${ }^{13}$ Over 2,500 patients were enrolled from 2013 through 2015 at participating hospitals, which included public and private nonprofit hospitals, across the four main counties in Hawaii (Maui County, Kauai County, Hawaii County, and Honolulu County). All members of the Hawaii Community Pharmacists Association, the association of independent pharmacies in the state, were invited but were not required to participate as community pharmacies. This care transition and care coordination model significantly reduced medication-related hospitalizations among older adults across these regions in Hawaii ${ }^{14}$ and was supported by technology that was implemented by Hawaii Health Information Exchange (HHIE), the only system in Hawaii with statewide coverage. The purpose of this article is to describe the technology that was implemented to support the pharmacists delivering the services in this model and to present the current status of the technology.

\section{Objective}

Our objective is to describe the design, implementation, and use of health IT to support hospital and community pharmacists as they manage medications for high-risk patients statewide in a new model of care called "Pharm2Pharm." This pharmacist-led service model reduced medication-related hospitalizations and achieved a robust return on investment in the pharmacists as previously reported. ${ }^{14}$ Throughout the design and implementation of the supporting technology, our primary aim was to make it easier for the pharmacists to access information needed to identify and resolve drug therapy problems using best practices for medication management. Our secondary aim was to make it easier for other members of the care team to access the results of the pharmacists' work. As we worked toward these aims, we were guided by two key principles:

1. The Pharm2Pharm model would be independent of and implemented before the supporting health IT. This ensured that the model itself could be replicated in any environment and that the health IT was designed based on experience with the model.

2. The health IT implemented to support the Pharm2Pharm model would add value to the healthcare system beyond that model to make the best use of the investment in health IT.

\section{Methods}

\section{Setting}

The technology described in this article supported the pharmacists providing services for the Pharm2Pharm model in the state of Hawaii, which included 50 pharmacists who were authorized by HHIE to use the technology. This includes the hospital pharmacists who enrolled patients into the Pharm2Pharm service as well as the community pharmacists 
who provided medication management services after discharge across the four counties in Hawaii which include both rural and urban settings. Founded in 2006, HHIE is the statedesignated entity for HIE in the state of Hawaii and also served as the Regional Extension Center for Hawaii.

\section{Study Design}

Qualitative and quantitative nonexperimental observational methods were used. An assessment of the pharmacists' technology needs was conducted based on their defined roles, medication management processes, and experience delivering the Pharm2Pharm services without supporting technology. This assessment guided the design of the supporting technology. The key Pharm2Pharm model roles and medication management processes are detailed in Standard Operating Procedures (available upon request) and summarized below.

\section{Hospital Pharmacist's Role}

- Identify and enroll patients at risk of medication problems according to evidence-based criteria.

- Manage medications during the hospital stay (as described below).

- Formally hand off the patient to the community pharmacist at discharge.

- Review readmissions of any previously enrolled patient as a feedback mechanism to continuously improve the model. ${ }^{15}$

\section{Community Pharmacist's Role}

- Manage medications (as described below) across prescribers and across dispensing pharmacies for up to a year postdischarge.

\section{Medication Management Processes}

All the hospital and community pharmacists who delivered the Pharm2Pharm services attended live training on the model, which included medication management processes adapted from existing best practice sources. ${ }^{16-19}$ Medication management in this model refers to the systematic process of ensuring that the patient's medication regimen is optimal with regard to appropriateness, effectiveness, and safety and that the patient is adhering to this regimen to promote health and reduce the need for acute care use. The medication management training has since been translated to an online, self-guided program "Identifying and Resolving Drug Therapy Problems across the Continuum of Care" (http:// pharmacy.uhh.hawaii.edu/ce/irdtp.php). The key steps of the medication management process, an iterative cycle of optimizing the drug therapy regimen, are as follows:

Medication reconciliation: The purpose of this step is to create a complete and accurate list of the patient's medications, including prescription and over-the-counter medications, vitamins, herbals, and other supplements. This requires accessing available medical records as well as interviewing the patient. A critical task in reconciling medications is resolving any discrepancies among the sources of information. Specifically, we adopted the definition of discrepancy as any lack of agreement between the medications in the patient's records and the patient's report of what he/she is taking. ${ }^{20}$ This includes inconsistencies in medications taken or not as well as in other aspects such as dose, frequency, or route.

Review of medical conditions: This step includes understanding the patient's current clinical status with regard to key health metrics (e.g., vital signs, laboratory results, etc.), the prescribers' clinical goals for the patient, as well as the patient's personal health goals. ${ }^{16}$

Addressing drug therapy problems: With the information from the previous two steps, drug therapy problems can be identified and resolved. This step involves first optimizing the medication regimen to achieve prescriber and patient goals, then working with the patient on adherence. ${ }^{16}$ Recommended solutions to key drug therapy problems are categorized as follows:

- Indication problems:

- Patient needs additional medication for an untreated condition.

- Medication should be discontinued because it is not indicated.

- Effectiveness problems:

- Dose should be increased because the medication is ineffective at the current dose.

- Medication should be replaced with another because it is not effective when taken at the recommended dose per guidelines.

- Safety/Side effect problems:

- Dose should be reduced.

- Medication should be replaced with another because the patient is experiencing (or is at unnecessary risk of experiencing) an adverse drug reaction or intolerable side effects.

- Adherence problems:

- Change the medication to one with simpler dosing.

- Assist the patient with use of a pill organizer and/or reminder system.

- Educate the patient regarding proper administration.

- Switch to a less expensive medication and/or refer to social services.

- Address cultural, personal, and/or communication issues that interfere with the patient taking the medication properly.

The Pharm2Pharm model, with the above roles and responsibilities, was launched and implemented for 7 months, spanning three counties, with very basic technology, specifically the hospital EMRs (with the exception of one hospital that was still using paper records at the time of launch), cell phones, and fax machines. During this time, the following needs were identified as the priorities to be addressed through health IT tools:

More efficient and secure way to transmit care transition documents: Using fax machines to send care transition documents, which were often dozens of pages, was cumbersome on both the sending and receiving end. The hospital pharmacists needed a secure and efficient method of sending hospital records and their handoff notes to the community 
pharmacists to support the smooth transition of medication management services at discharge.

Reliable access to outpatient translators: In Hawaii, approximately one-fourth of the population speaks a language other than English at home. ${ }^{21}$ There are dozens of different languages spoken across the state, and the counties differ with regard to the most common non-English language spoken at home. For example, the top Honolulu County language is Tagalog (one of the Filipino languages), Hawaii County is Hawaiian language, and Maui County is Ilocano (one of the Filipino languages). While all of the hospitals have virtual translation services to accommodate this diverse population, the hospital pharmacists could not enroll non-English speaking patients in the Pharm2Pharm model without the availability of outpatient translation services. Even for patients with family members willing to translate, research shows that use of professional translators is associated with better patient care compared with ad hoc translators and results in quality care equivalent to those without language barriers.22

More efficient way to identify outpatient medications for medication reconciliation: While the hospital pharmacists had access to the inpatient medications, the patients' reports of their home medications collected upon admission is very often incomplete or incorrect. Previous research indicates that about half of all general medicine inpatients have unexplained discrepancies between their preadmission medications and discharge medications. ${ }^{23}$ The community pharmacists also had challenges reconciling medications. While they had access to the patients' medications that were dispensed at their specific pharmacy where they worked, many patients used a different pharmacy or multiple pharmacies to purchase their medications. Thus, both hospital and community pharmacists had to rely predominantly on the patient report of medications when reconciling medications as well as phone calls to pharmacies and ambulatory clinics to resolve any discrepancies.

More efficient access to clinical information: The community pharmacists needed more timely and efficient access to their patients' medical records. They needed access to hospital records beyond the care transition documents received from the hospital pharmacists, particularly information regarding subsequent emergency department (ED) visits and hospitalizations. They also needed access to outpatient records, particularly laboratory results, to support review of the status of medical conditions. At the time of launch, this required requesting records to be faxed from the patient's primary care provider and specialists. Access to clinical information is essential in performing best-practice medication management, and lack of access has been a barrier for pharmacists performing medication therapy management (MTM) under a Medicare Part D plan. ${ }^{24,25}$ With MTM under Part D, the pharmacists typically have little or no clinical information about the patient. This means the pharmacists are limited in their ability to help optimize medication regimens and tend to focus on adherence (without knowing if the medication is indicated or effective) and safety issues (such as drug-drug interactions) based on the Part D prescription claims information. Lack of integration with the healthcare team, including lack of access to medical records, has been identified by community pharmacists as the most serious barrier to improving health outcomes through MTM services, ${ }^{25}$ and is likely a key reason why physicians have been reluctant to accept recommendations from pharmacists providing MTM under Part D. ${ }^{26}$ This clinical information gap might also be one of the reasons a systematic review and meta-analysis was inconclusive regarding the effect of MTM interventions on most of the outcomes examined. ${ }^{27}$ With access to clinical information, the pharmacist can first work with the patients and prescribers to optimize the medication regimen (i.e., by identifying and resolving indication problems, effectiveness problems, and safety/side effect problems) and then work with the patient to ensure adherence to this regimen. This is important because previous research has shown that adherence problems comprise only a minority of drug therapy problems, while indication or effectiveness problems are the most common. ${ }^{17-19}$

System to document and communicate reconciled medication list and drug therapy problems: The pharmacists each maintained their patients' records of the current reconciled medication list and drug therapy problems. They also communicated individually with relevant prescribers. However, these and other clinicians did not have automatic access to the pharmacist's updated reconciled medication list or drug therapy problems.

System to manage the population of patients enrolled: In the traditional dispensing role, pharmacists manage prescriptions, not patients. In the Pharm2Pharm model, community pharmacists were responsible for managing a population of high-risk patients postdischarge. As their patient load grew, keeping track of patients became increasingly challenging. An effective system was needed to support the proactive approach of panel or population management. ${ }^{28}$ Previous research has shown that electronic medical record tools can improve population management in primary care practices, ${ }^{29}$ but the community pharmacists working across primary care practices and specialists in the Pharm2Pharm model did not have such tools.

Mobility: While not required in the Pharm2Pharm model, some of the community pharmacists offered home visits. In one case, a pharmacist conducted visits while a patient was receiving dialysis to optimize convenience for the patient. Whether moving between offices at the pharmacy or doing off-sites visits, the pharmacists needed to be mobile with their patients' records.

This assessment was the basis for designing solutions. During the implementation of the solutions, key milestones and processes were documented. Finally, frequency of use was tracked postimplementation.

\section{Results}

With these priority needs identified, technology tools were designed and implemented as summarized in -Table 1.

The tools, categorized as addressing communications, medication management, and population management, are shown in - Fig. 1, and the implementation and utilization of each are described below. In addition to these tools, the pharmacists were given portable Mi-Fi devices (mobile wireless internet 
Table 1 Tools to address the identified priority needs

\begin{tabular}{|l|l|}
\hline Priority needs & Technology tools \\
\hline $\begin{array}{l}\text { More efficient and secure way to transmit } \\
\text { care transition documents }\end{array}$ & HHIE direct secure messaging \\
\hline Reliable access to outpatient translators & LAN virtual translators \\
\hline $\begin{array}{l}\text { More efficient way to identify outpatient medications } \\
\text { for medication reconciliation }\end{array}$ & HCS prescription fill history query tool \\
\hline More efficient access to clinical information & $\begin{array}{l}\text { HHIE community health record with hospital information } \\
\text { and outpatient laboratory interfaces }\end{array}$ \\
\hline $\begin{array}{l}\text { System to document and communicate reconciled } \\
\text { medication list and drug therapy problems }\end{array}$ & $\begin{array}{l}\text { HCS medication reconciliation documentation tool and drug } \\
\text { therapy problem documentation tool; documentation interface } \\
\text { with the HHIE community health record }\end{array}$ \\
\hline System to manage the population of patients enrolled & HHIE patient referral and registry tools \\
\hline Mobility & Ultra-light laptops and wireless internet access devices \\
\hline
\end{tabular}

Abbreviations: HCS, Health Care Systems; HHIE, Hawaii Health Information Exchange; LAN, Language Access Network.

access) and ultra-light laptops configured to easily access the solutions from any location with cell reception. The pharmacists in the first three counties where Pharm2Pharm had been implemented made the transition from paper and faxes to this suite of technology. In the last county, most of this technology was available at the time of launch so that the pharmacists did not have to make this transition.

HHIE direct secure messaging: HHIE launched direct secure messaging to clinicians in Hawaii in 2012; so, this functionality was in place before the launch of Pharm2Pharm and its utilization has varied along with changes in Meaningful Use requirements. In October 2013, the hospital pharmacist at one Pharm2Pharm-enrolling hospital and the community pharmacists in that region were given access to the existing HHIE direct secure messaging system to pilot its use for transmitting care transition documents. This pilot confirmed the feasibility of secure messaging for this use case; so, it was then rolled out to the other hospital and community pharmacists. Over a 1-year period, the care transition documents sent from hospital to community pharmacist shifted from $100 \%$ fax to $100 \%$ direct secure messaging via HHIE. After this milestone was achieved, efforts were made to identify physicians who would prefer to receive communications from the pharmacists via secure messaging rather than fax. In 2014 and 2015, between 4 and $5 \%$ of physicians with patients enrolled in Pharm2Pharm were using HHIE secure messaging for written communications. In 2016, that number increased to $8 \%$. As an indicator of the extent to which the Pharm2Pharm program was driving utilization of this technology, in all but 1 month over a 1 -year period, the number of secure messages sent by a Pharm2Pharm

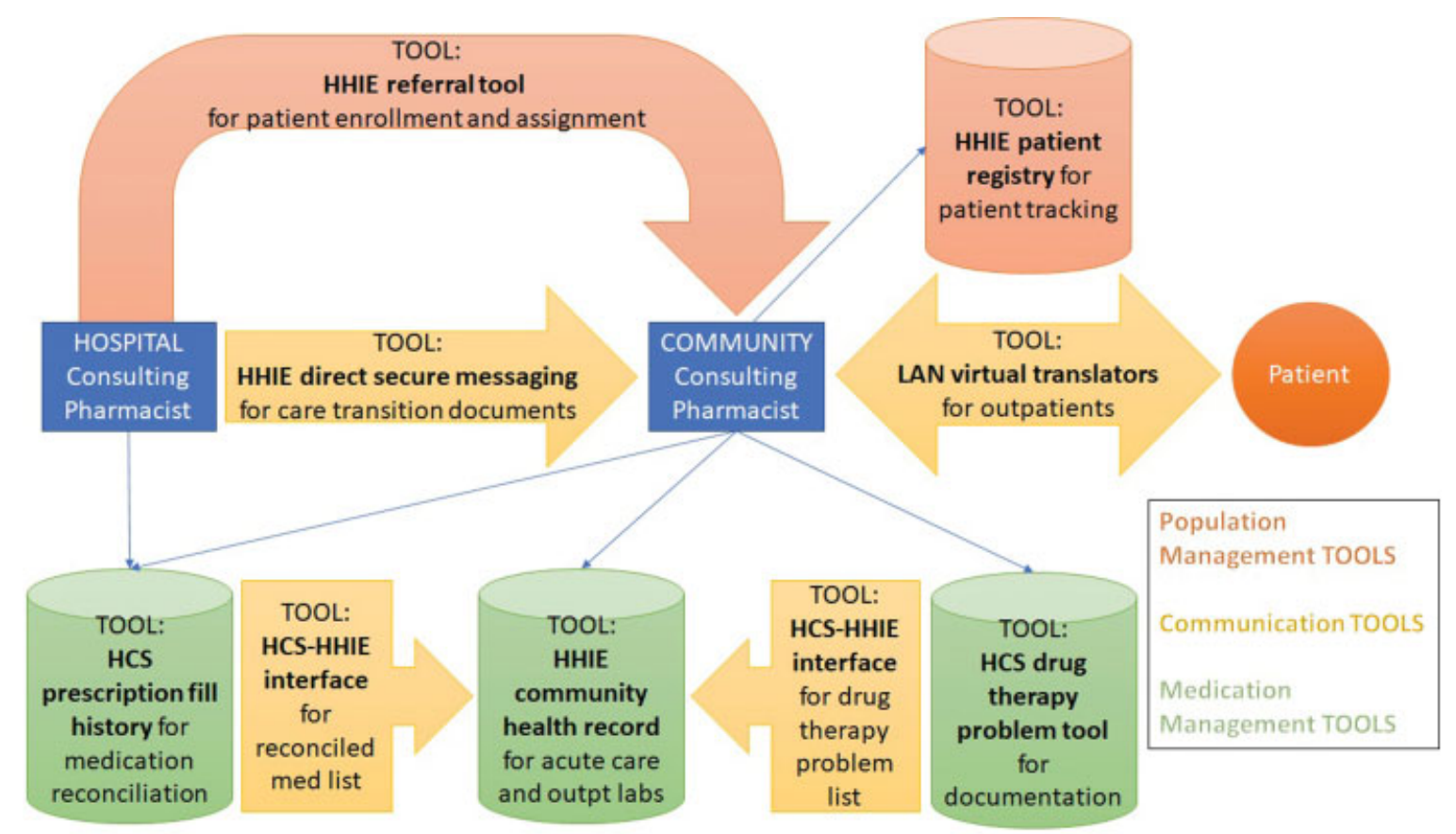

Fig. 1 Communication, medication management, and population management tools. 


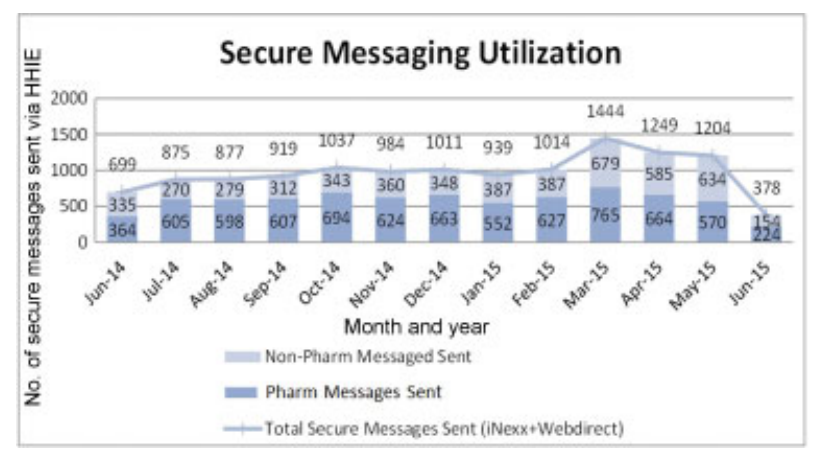

Fig. 2 Total number of secure messages sent by group: pharmacist versus other.

pharmacist was greater than the number of messages sent by all other users as shown in - Fig. 2. This figure also shows a decrease in pharmacist use as enrollment was winding down in June of 2015. In addition, other users decreased in June as HHIE rolled out a referral module that replaced some use of direct secure messaging and as two high-utilizing organizations developed in-house ability to send secure messages within their respective organizations. There has been a slow but steady increase in the use of direct secure messaging since June 2015 among organizations that have internal secure messaging systems but use HHIE as their health information service provider.

HCS prescription fill history and medication reconciliation documentation: Health Care Systems (HCS) prescription fill history tool is a query system that supports medication reconciliation by giving the pharmacist, within seconds, a list of the patient's medications that have been filled within the past 12 months. This tool was piloted with live patient data beginning in February 2014 and was found to produce a robust and complete history with the large majority of patients. By June of 2014, all of the pharmacists had been trained on the system and were using it as part of the medication reconciliation process. As shown in -Fig. 3 , the HCS tool had prescription fill history results on $90 \%$ of queries. At the time of launch, the only prescriptions not included in this query system were those filled by Kaiser or the Veterans Affairs (VA) and those paid with cash with no claim filed. More recently, the prescriptions filled at VA and Department of Defense clinics have been added to the HCS system, making this an even more valuable query tool. By the end of 2014, this prescription fill history query function was

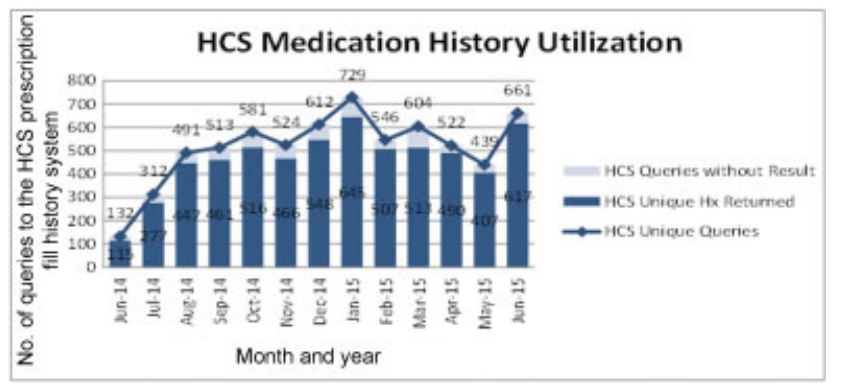

Fig. 3 Total number of HCS prescription fill history queries. integrated into the community health record. In addition to showing the fill history, the HCS system allows the pharmacist to add to the list, including over-the-counter medications, vitamins, herbals, and other supplements. The result is a document with the pharmacist's complete and accurate medication list. In November 2015, the Consolidated Clinical Document Architecture integration was completed so that this reconciled medication list document is transmitted to the community health record for other clinicians to use rather than starting from scratch.

LAN virtual translators: In June 2014, each community pharmacist was given phone access to Language Access Network (LAN) virtual translators who covered all of the many Asian and Pacific languages regularly spoken in Hawaii as well as many more. That same month, the first patient was enrolled who previously would not have been due to language barriers. Over a 2-month period of tracking, there were 25 community pharmacist calls to translators with a total of 232 minutes of talk time in the following languages: Chuukese, Hmong, Japanese, Spanish, Cantonese, Ilocano, and Mandarin.

Patient referral and registry modules: These modules were piloted beginning in June 2014. The existing referral system was modified to be used by the hospital pharmacists at the time of enrollment to refer or assign each patient to a specific community pharmacist. The patient registry module was then used by the pharmacists to manage the population. It was this registry system that documented the authority of the pharmacists to access the patient's community health record. By December 2014 , all hospital pharmacists were using the system to enroll patients, and the community pharmacists were then able to use the system to track each patient through program exit. One request by the pharmacists was to have a patient scheduling tool to remind them when each patient was due for a visit, based on the frequency set at the previous visit, and to schedule the visit. Because this functionality was not available, HHIE developed an Excel tool to perform this function. Ideally such a tool would be integrated into the system used for tracking patients.

HHIE community health record: At the time of Pharm2Pharm launch, a multisystem integrated community health record was not yet functional. Over time, interfaces between two key sources of data were completed, providing the pharmacists and other clinicians with important health information consolidated into one view

Hospital interfaces: In November 2013, the first interface was built between one of the hospitals participating in the Pharm2Pharm program and the HHIE. The data became available through the community health record in March of 2015, and other hospitals across the state were subsequently added. These interfaces include real-time information about ED visits and hospitalizations.

Laboratory interfaces: By early 2014, laboratory interfaces were completed between the HHIE community health record and the two laboratory companies processing nearly $100 \%$ of all outpatient laboratory results in Hawaii. At that time, state administrative rule prohibited pharmacists from having direct access to a patient's laboratory results unless the ordering physician gave written authorization. Therefore, the laboratory representatives, who regularly visited physician practices, were 
trained to explain the Pharm2Pharm model and ask the physicians to authorize pharmacist access. As of November 2014, a total of 185 physicians, the majority of those contacted, had authorized pharmacist access to the laboratory results. By that time, revised Clinical Laboratories Improvement Act (CLIA) regulations superseded state rule by allowing patients to authorize who has access to their laboratory results. This revision also clarified that "HIPAA covered entities that are in a treatment relationship with the individual" are among those "responsible for using the test results" to whom laboratory results may be released. ${ }^{30}$ Since 2012 , HHIE had been actively engaging CLIA staff at the local and national level to ensure appropriate access to test results, and the local CLIA representative was supportive in guiding HHIE in establishing procedures for pharmacists to have access to their Pharm2Pharm patients' laboratory results.

HCS drug therapy problem tool: By June 2015, a module was built to document drug therapy problem identification and resolution leveraging the HCS medication reconciliation module. This tool was custom developed for the Pharm2Pharm model to allow the pharmacists to document their systematic process of identifying drug therapy problems. This tool used the prepopulated medications from the reconciled medication list in the HCS system. The drug therapy problem list from this technology was being built to be interfaced to the community health record. However, this interface was not completed. This customized tool is the only solution that was developed specifically for Pharm2Pharm. Its use did not continue after the program funding ended.

As the Pharm2Pharm program funding was winding down, several pharmacists participated in pilot use of the HHIE "clinical inbox" functionality, which integrates secure messaging, the community health record, and the patient registry (see screen shot in -Fig. 4). This function allows customized alerts, flagged as clinically important, to be delivered to the clinician's HHIE inbox. For example, outpatient clinicians can have a secure email automatically sent to their clinical inbox when one of their patients has been admitted to the hospital or has a new laboratory result. The pharmacists found this tool to be valuable, and this has become one of the more frequently used tools among HHIE users statewide.

Across these health IT tools, a recurrent barrier to adoption was the lack of experience and comfort with technology among some of the community pharmacists. Given that pharmacists were not included as eligible providers under Meaningful Use programs, many are not familiar with technologies such as electronic medical records and secure messaging that are routinely used by other clinicians. This barrier was addressed with a combination of training and staff support. All pharmacists completed the technology training. Those with additional needs were given individual technology support, resulting in adoption among all the pharmacists.

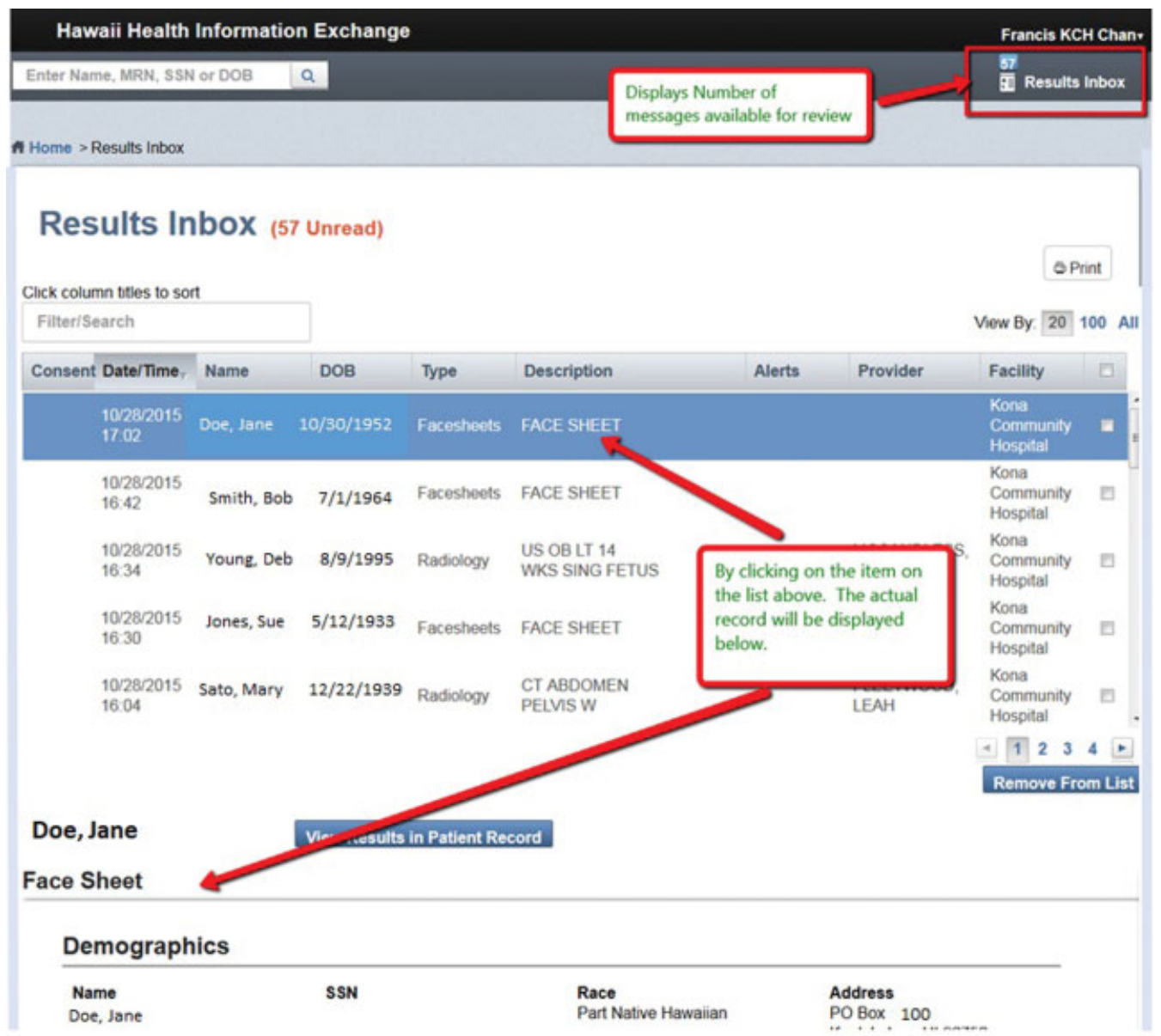

Fig. 4 Screenshot of the HHIE clinical inbox tool. 


\section{Discussion}

Pharmacists adopted and meaningfully used this suite of health IT to improve the efficiency and effectiveness of communications, medication management, and population management delivered through the Pharm2Pharm model. We believe this represents the first statewide integration of pharmacists into a health information exchange and the first time a prescription fill history query system has been integrated into an HIE. As previously reported, the Pharm2Pharm model was associated with a significant reduction in medication-related hospitalizations and a $264 \%$ return on the investment in the pharmacist services. $^{14}$ The estimated annual net cost savings from the Pharm2Pharm model was significantly greater than the total cost of implementing the health IT, given that the HHIE infrastructure and basic functionality was already in place. Thus, the Pharm2Pharm model and the supporting technology described in this article should be considered a robust approach to improving patient care and reducing costs, though the independent contribution of the health IT to improving the efficiency or effectiveness of the model was not tested. The technology used in the Pharm2Pharm model also demonstrates the importance of having health information exchange infrastructure to support population health interventions, and confirms the importance of ensuring such infrastructure is built to support users' information needs and workflow. ${ }^{31}$

From a tactical perspective, this work provides several useful lessons. First, there is benefit to conceptualizing new models of care independent of technology. The Pharm2Pharm model was designed and implemented before the technology solutions. This means that the model can be replicated in other environments that do not have these solutions in place. This also means that the technology priorities identified in this project could be addressed with other solutions. Second, this work emphasizes the importance of shifting the focus of medication management technology from supporting a specific piece of the process, such as e-prescribing or medication reconciliation, to supporting the goal of optimizing medication regimens, particularly for complex patients who are at increased risk of medication problems. Finally, it is noteworthy that, even after the funding for the Pharm2Pharm model ended, most of the technology described here is still in use. This is because of the value placed on investing in technology that had solid use cases beyond Pharm2Pharm. For example, secure messaging and central access to clinical information such as acute care use, laboratory results, and prescription medication fill history are important tools for nurses and physicians who are involved in care transitions and managing populations at risk. As previously reported, ${ }^{14}$ sustaining the pharmacist services beyond the Pharm2Pharm award funding is challenging due to current payment mechanisms, despite a robust return on investment. Because most of the technology funded through the award was already on the list of HHIE priorities for roll out, its use among other clinicians beyond the award period continues. As payment for pharmacists' clinical services (vs. dispensing services) is addressed, this work demonstrates that HIE systems are ideal for reducing the fragmentation among pharmacists working in hospital and community settings.

\section{Conclusion}

Pharmacists have unique expertise in optimizing drug therapy, an emerging role distinct from the traditional drug dispensing role of the pharmacist. While there is increasing evidence of the positive impact of pharmacists deployed as medication experts, health IT is generally not available, outside of integrated healthcare systems, to support pharmacists in this role. Access to medical records, a key challenge that prevents the integration of pharmacists into care teams, ${ }^{32}$ is critical for pharmacists to identify and resolve the most common drug therapy problems. Health information exchange systems, as demonstrated by HHIE and the pharmacists who adopted technology for the Pharm2Pharm model, are uniquely positioned to provide the technology pharmacists need to support their integration into care teams and best practice medication management to improve patient outcomes.

\section{Clinical Relevance Statement}

We reported on the use of health IT by hospital and community pharmacists providing medication management services in an innovative model of care. Our findings suggest that HIE technology is a valuable approach to support the integration of pharmacists into care teams. More research is needed to quantify the unique impact of health IT on improving the quality and efficiency of pharmacists working in clinical roles.

\section{Multiple Choice Questions}

1. To identify all types of drug therapy problems, what information do pharmacists need?

a. The patient's medications

b. The patient's medical conditions

c. Laboratory results related to the patient's medical conditions

d. All of the above

Correct Answer: The correct answer is d, All of the above. Pharmacists need to know the patient's medications to evaluate them for problems. Pharmacists need to know the patient's medical conditions to identify indication problems, specifically untreated indications or unnecessary medications. Pharmacists need the laboratory results related to the patient's medical conditions so that they can identify effectiveness and safety problems.

2. Which key barrier can health information exchange systems help address to improve health outcomes through medication management services provided by community pharmacists?

a. Lack of payment for pharmacist services

b. Lack of pharmacist's integration with the healthcare team

c. Lack of pharmacist training

d. None of the above 
Correct Answer: The correct answer is b, Lack of pharmacist's integration with the healthcare team. While payment for pharmacists' clinical services and pharmacist training in standardized approaches are barriers to improving health outcomes through medication management, the barrier that can be addressed through HIE technology is lack of integration into care teams. HIE technology makes it easier for the pharmacists to access information needed to identify and resolve drug therapy problems and makes it easier for other members of the care team to access the results of the pharmacists' work. This improved information access across clinicians and healthcare organizations is the core purpose of HIE technology.

\section{Protection of Human and Animal Subjects}

This study was approved as exempt by the University of Hawaii Institutional Review Board.

\section{Funding}

The project described was supported by Funding Opportunity number CMS-1C1-12-0001 from Centers for Medicare and Medicaid Services, Center for Medicare and Medicaid Innovation. Its contents are solely the responsibility of the authors and do not necessarily represent the official views of HHS or any of its agencies.

\section{Conflict of Interest}

None.

\section{Acknowledgments}

We would like to acknowledge the consulting pharmacists who adopted and meaningfully used the technology described in this article; the technology and support teams at Hawaii Health Information Exchange, Health Care Systems, and Language Access Network; and Christine Mai'i Sakuda, founding Executive Director of Hawaii Health Information Exchange, for her leadership and dedication to improving health in Hawaii.

\section{References}

1 Centers for Medicare and Medicaid Services. Available at: https:// questions.cms.gov/faq.php?id=5005\&faqId=2939. Accessed May 4, 2017

2 Furukawa MF, Spector WD, Rhona Limcangco M, Encinosa WE. Meaningful use of health information technology and declines in in-hospital adverse drug events. J Am Med Inform Assoc 2017;24 (04):729-736

3 Gabriel MH, Smith JY, Sow M, Charles D, Joseph S, Wilkins TL. Dispatch from the non-HITECH-incented Health IT world: electronic medication history adoption and utilization. J Am Med Inform Assoc 2016;23(03):562-569

4 McKibbon KA, Lokker C, Handler SM, et al. The effectiveness of integrated health information technologies across the phases of medication management: a systematic review of randomized controlled trials. J Am Med Inform Assoc 2012;19(01):22-30

5 Marien S, Krug B, Spinewine A. Electronic tools to support medication reconciliation: a systematic review. J Am Med Inform Assoc 2017;24(01):227-240
6 Schnipper JL, Hamann C, Ndumele CD, et al. Effect of an electronic medication reconciliation application and process redesign on potential adverse drug events: a cluster-randomized trial. Arch Intern Med 2009;169(08):771-780

7 Nabovati E, Vakili-Arki H, Taherzadeh Z, et al. Information technology-based interventions to improve drug-drug interaction outcomes: a systematic review on features and effects. J Med Syst 2017;41(01):12

8 Joint Commission of Pharmacy Practitioners. Pharmacists' Patient Care Processes, May 29, 2014; Available at: https://www.pharmacist.com/sites/default/files/files/PatientCareProcess.pdf. Accessed May 4, 2017

9 Nelson SD, Poikonen J, Reese T, El Halta D, Weir C. The pharmacist and the EHR. J Am Med Inform Assoc 2017;24(01):193-197

10 Chisholm-Burns MA, Kim Lee J, Spivey CA, et al. US pharmacists' effect as team members on patient care: systematic review and meta-analyses. Med Care 2010;48(10):923-933

11 Lee JK, Slack MK, Martin J, Ehrman C, Chisholm-Burns M. Geriatric patient care by U.S. pharmacists in healthcare teams: systematic review and meta-analyses. J Am Geriatr Soc 2013;61 (07):1119-1127

12 Pellegrin KL, Miyamura J, Taniguchi R, Ciarleglio AE, Barbato A, Scott Holuby R. Using International Classification of Diseases Codes to track medication-related hospitalizations of older adults. J Am Geriatr Soc 2016;64(03):651-653

13 Pellegrin KL. The Daniel K. Inouye College of Pharmacy Scripts: Pharm2Pharm: Leveraging medication expertise across the continuum of care. Hawaii J Med Public Health 2015;74(07): 248-252

14 Pellegrin KL, Krenk L, Oakes SJ, et al. Reductions in medicationrelated hospitalizations among older adults with medication management by hospital and community pharmacists: a quasiexperimental study. J Am Geriatr Soc 2017;65(01):212-219

15 Pellegrin KL, Lee E, Uyeno R, Ayson C, Goo R. Potentially preventable medication-related hospitalizations: a clinical pharmacist approach to assessment, categorization, and quality improvement. J Am Pharm Assoc (2003) 2017;57(06):711-716

16 Cipolle RJ, Strand LM, Morley PC. Pharmaceutical Care Practice The Patient Centered Approach to Medication Management. 3rd ed. New York, NY: McGraw Hill; 2012

17 Isetts BJ, Schondelmeyer SW, Artz MB, et al. Clinical and economic outcomes of medication therapy management services: the Minnesota experience.J Am Pharm Assoc (2003) 2008;48(02):203-211, 3 p following 211

18 Ramalho de Oliveira D, Brummel AR, Miller DB. Medication therapy management: 10 years of experience in a large integrated health care system. J Manag Care Pharm 2010;16(03):185-195

19 Smith M, Giuliano MR, Starkowski MP. In Connecticut: improving patient medication management in primary care. Health Aff (Millwood) 2011;30(04):646-654

20 Orrico KB. Sources and types of discrepancies between electronic medical records and actual outpatient medication use. J Manag Care Pharm 2008;14(07):626-631

21 Hawaii State Data Center. Detailed Languages Spoken at Home in the State Hawaii. Available at: http://files.hawaii.gov/dbedt/ census/acs/Report/Detailed_Language_March2016.pdf. Accessed May 4, 2017

22 Karliner LS, Jacobs EA, Chen AH, Mutha S. Do professional interpreters improve clinical care for patients with limited English proficiency? A systematic review of the literature. Health Serv Res 2007;42(02):727-754

23 Schnipper JL, Kirwin JL, Cotugno MC, et al. Role of pharmacist counseling in preventing adverse drug events after hospitalization. Arch Intern Med 2006;166(05):565-571

24 Vora J. Access to a health system's electronic medical record. Pharmacy Times September 2015. Available at: http://www.pharmacytimes.com/publications/health-system-edition/2015/september2015/access-to-a-health-systems-electronic-medical-record 
10 Health Information Exchange to Support Drug Therapy Optimization Pellegrin et al.

25 Smith MA, Spiggle S, McConnell B. Strategies for communitybased medication management services in value-based health plans. Res Social Adm Pharm 2017;13(01):48-62

26 Medicare Payment Advisory Commission. "Medication therapy management programs under Part D". Available at: http://www. medpac.gov/docs/default-source/comment-letters/022 82014_par td_comment.pdf?sfvrsn=0. Accessed May 4, 2017

27 Viswanathan M, Kahwati LC, Golin CE, et al. Medication therapy management interventions in outpatient settings: a systematic review and meta-analysis. JAMA Intern Med 2015;175(01): $76-87$

28 US Department of Health and Human Services, Agency for Healthcare Research and Quality. Available at: https://www.ahrq.gov/ professionals/prevention-chronic-care/improve/system/pfhandbook/mod20.html. Accessed May 4, 2017
29 Loo TS, Davis RB, Lipsitz LA, et al. Electronic medical record reminders and panel management to improve primary care of elderly patients. Arch Intern Med 2011;171(17):1552-1558

30 US Office of the Federal Register. Available at: https://www. federalregister.gov/documents/2014/02/06/2014-02280/clia-pro gram-and-hipaa-privacy-rule-patients-access-to-test-reports. Accessed May 4, 2017

31 Kierkegaard P, Kaushal R, Vest JR. How could health information exchange better meet the needs of care practitioners? Appl Clin Inform 2014;5(04):861-877

32 Joseph T, Hale GM, Eltaki SM, et al. Integration strategies of pharmacists in primary care-based accountable care organizations: a report from the Accountable Care Organization Research Network, Services, and Education. J Manag Care Spec Pharm 2017; 23(05):541-548 